\title{
Revascularization of the anterior cerebral artery by Y-shaped superficial temporal artery interposition graft for the treatment of a de novo aneurysm arising at the site of $A_{3}-A_{3}$ bypass: technical case report
}

\author{
Hidenori Endo, MD, PhD, ${ }^{1}$ Shin-ichiro Sugiyama, MD, PhD, ${ }^{2}$ Toshiki Endo, MD, PhD, ${ }^{1}$ \\ Miki Fujimura, MD, PhD, ${ }^{3}$ Hiroaki Shimizu, MD, PhD, ${ }^{4}$ and Teiji Tominaga, MD, $\mathrm{PhD}^{3}$

\begin{abstract}
Departments of ${ }^{1}$ Neurosurgery and ${ }^{2}$ Neuroanesthesia, Kohnan Hospital, Sendai; ${ }^{3}$ Department of Neurosurgery, Tohoku University Graduate School of Medicine, Sendai; and ${ }^{4}$ Department of Neurosurgery, Akita University Graduate School of Medicine, Akita, Japan
\end{abstract}

\begin{abstract}
The most frequently used option to reconstruct the anterior cerebral artery (ACA) is an ACA-ACA side-to-side anastomosis. The long-term outcome and complications of this technique are unclear. The authors report a case of a de novo aneurysm arising at the site of $A_{3}-A_{3}$ anastomosis. A 53-year-old woman underwent $A_{3}-A_{3}$ side-to-side anastomosis for the treatment of a ruptured right $A_{2}$ dissecting aneurysm. At 44 months after surgery, a de novo aneurysm developed at the site of anastomosis. The aneurysm developed in the front wall of the anastomosis site, and projected to the anterosuperior direction. A computational fluid dynamics (CFD) study showed the localized region with high wall shear stress coincident with the pulsation in the front wall of the anastomosis site, where the aneurysm developed. A Y-shaped superficial temporal artery (STA) interposition graft was used successfully to reconstruct both ACAs, and then the aneurysm was trapped. To the authors' knowledge, this is the first case of a de novo aneurysm that developed at the site of an ACA-ACA side-to-side anastomosis. A CFD study showed that hemodynamic stress might be an underlying cause of the aneurysm formation. A Y-shaped STA interposition graft is a useful option to treat this aneurysm. Long-term follow-up is necessary to detect this rare complication after ACA-ACA anastomosis.
\end{abstract}

https://thejns.org/doi/abs/10.3171/2017.6.JNS17931

KEY WORDS de novo aneurysm; bypass surgery; anterior cerebral artery; interposition graft; vascular disorders

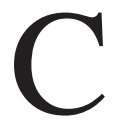
LIPPING and coiling are not applicable for complex intracranial aneurysms, such as giant, thrombosed, fusiform, or dissecting aneurysms. Trapping with revascularization is a therapeutic option for these types of aneurysms. For anterior cerebral artery (ACA) aneurysms, revascularization techniques are much more complicated than other techniques because of their deep location in the interhemispheric fissure.,19 There are many technical options and variations for revascularization of ACAs. ${ }^{1,8,14,19,23}$ The most reasonable and simplest option to reconstruct one side of the ACA is an ACA-ACA side-to-side anastomosis. ${ }^{9}$ This bypass technique was classified as in situ bypass or communicating bypass, and has been used most frequently in the recent literature. ${ }^{1,19}$ However, its longterm results and complications remain unclear. We report a case of a de novo aneurysm arising at a site of $A_{3}-A_{3}$ anastomosis, treated successfully by a Y-shaped superficial temporal artery (STA) interposition graft bypass. We also discuss the underlying cause of aneurysm formation using computational fluid dynamics (CFD) technology.

\section{Case Report}

History

A 53-year-old woman first presented with a sudden onset of severe headache. Computed tomography showed subarachnoid hemorrhage with intracerebral hematoma in the right rectal gyrus (Fig. 1A). Digital subtraction angiography (DSA) showed a growing aneurysm of the proximal $\mathrm{A}_{2}$ segment of the right ACA (Fig. 1B and C). The diagnosis was a dissecting aneurysm of the right $\mathrm{A}_{2}$ segment. An $\mathrm{A}_{3}-\mathrm{A}_{3}$ side-to-side anastomosis was performed (Fig.

ABBREVIATIONS ACA = anterior cerebral artery; $C F D=$ computational fluid dynamics; DSA = digital subtraction angiography; $\mathrm{MCA}=$ middle cerebral artery; $\mathrm{STA}=$ superficial temporal artery.

SUBMITTED April 13,2017. ACCEPTED June 28, 2017.

INCLUDE WHEN CITING Published online December 22, 2017; DOI: 10.3171/2017.6.JNS17931. 

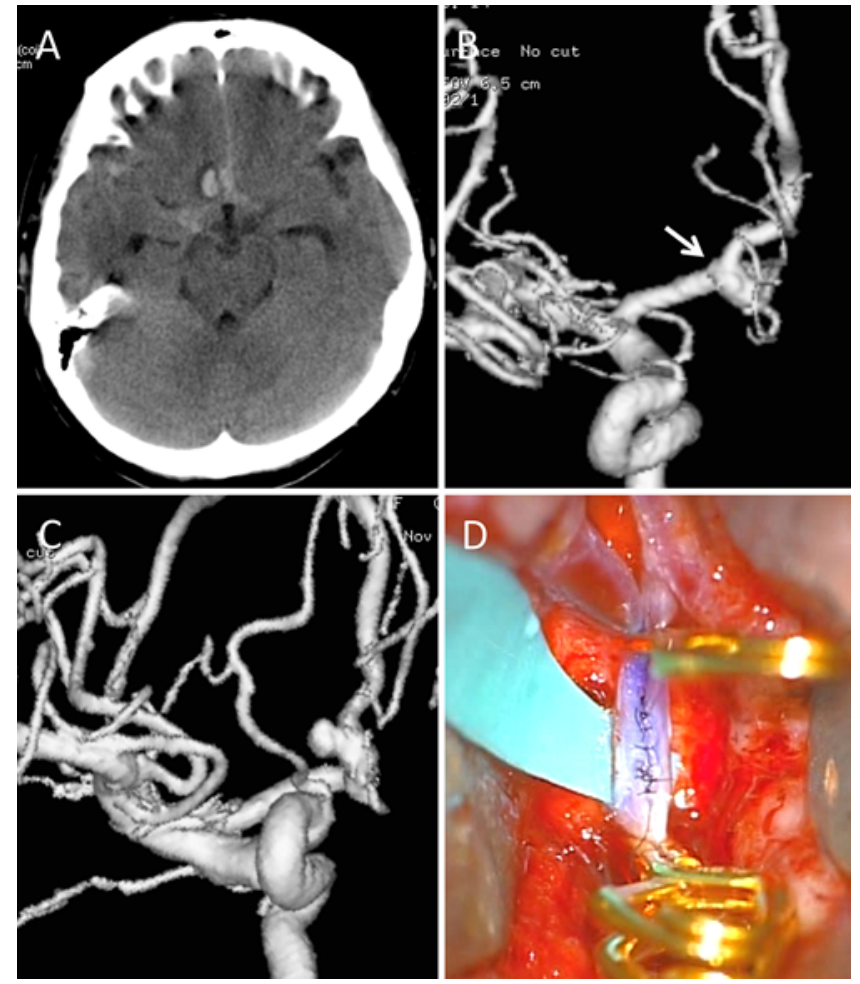

FIG. 1. A: Axial CT scan shows a subarachnoid hemorrhage with an intracerebral hematoma in the right rectal gyrus. B: Cerebral angiography shows the small bulge at the right proximal $\mathrm{A}_{2}$ segment (arrow). C: Follow-up angiography 9 days after the onset shows growth of the aneurysm. D: Intraoperative photograph shows the $A_{3}-A_{3}$ bypass. Figure is available in color online only.

1D), and the aneurysm was trapped and resected to prevent rebleeding. The histological diagnosis was a dissecting aneurysm (data not shown). Postoperative angiography showed disappearance of the dissecting aneurysm and patency of the $\mathrm{A}_{3}-\mathrm{A}_{3}$ bypass (Fig. 2A). The patient recovered and was discharged without any neurological deficits.
However, a de novo aneurysm was suspected at the site of the $\mathrm{A}_{3}-\mathrm{A}_{3}$ anastomosis by an annual MRI follow-up examination 44 months after surgery.

\section{Examination}

Aneurysm formation was not apparent on the first DS angiogram 14 days postoperatively (Fig. 2A). However, the existence of a small bulge was suspected on the second angiogram 6 months after surgery (Fig. 2B). This bulge apparently had grown to $5 \mathrm{~mm}$ in maximum diameter on the third angiogram 44 months postoperatively (Fig. 2C). A third DS angiogram showed that the blood flow from the left $\mathrm{A}_{2}$ segment turned back to the right proximal $\mathrm{A}_{2}$ segment, which hit against the front wall of the anastomosis site (Fig. 3A). Furthermore, a CFD study showed the localized region with high wall shear stress coincident with the pulsation in the front wall of the anastomosis site, where the aneurysm developed (Fig. 3B, Video 1).

VIDEO 1. Clip showing CFD study of the localized region with high wall shear stress (red) coincident with the pulsation in the front wall of the anastomosis site. Copyright Hidenori Endo. Published with permission. Click here to view.

Surgical obliteration of the growing aneurysm was scheduled to prevent possible risk for future rupture.

\section{Operation}

A curved bicoronal skin incision was made. On the right side, the skin incision was made along with the parietal branch of the right STA. The STA trunk and its branches (frontal and parietal) were dissected carefully as the skin flap was retracted. After removing the periadventitial tissue, a Y-shaped STA graft was harvested and immediately flushed with heparinized saline. Bifrontal craniotomy was then performed. The extent of the craniotomy was larger than during the first surgery so as to provide a wider opening of the interhemispheric fissure. After sharp dissection of the interhemispheric fissure, we exposed the aneurysm and the bilateral ACAs (Fig. 4A). The aneurysm developed in the front wall of the anastomosis site, and projected to
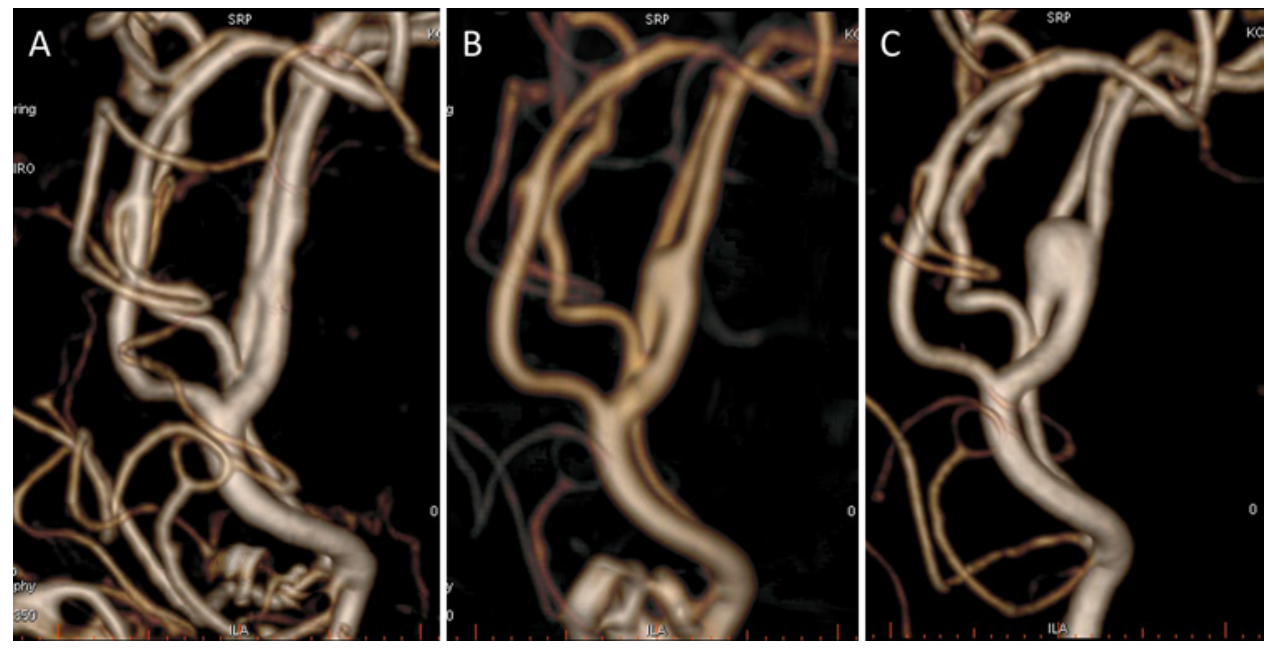

FIG. 2. A: Cerebral angiography obtained 14 days after surgery shows the disappearance of the right $A_{2}$ dissecting aneurysm and patency of the bypass. B: Follow-up angiography obtained 6 months after surgery shows a small bulge at the site of anastomosis. C: Follow-up angiography obtained 44 months postoperatively shows that the bulge had grown up to $5 \mathrm{~mm}$ in maximum diameter. Figure is available in color online only. 


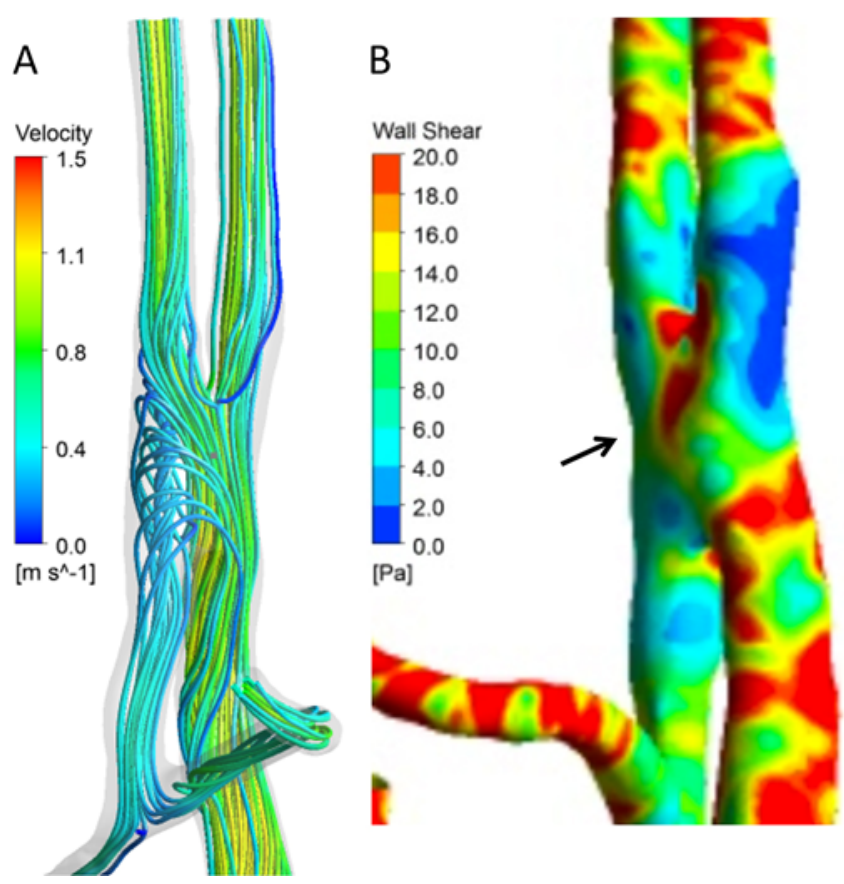

FIG. 3. A: CFD study performed before the aneurysm formation shows the hairpin-shaped blood stream at the site of anastomosis. B: CFD study shows the localized region with high wall shear stress coincident with the pulsation in the front wall of the anastomosis site (arrow). Figure is available in color online only.

the anterosuperior direction. Sutures had been placed in the anterior wall of the aneurysm during the previous surgery. The aneurysm appeared similar to the usual saccular aneurysms.

The frontal and parietal branches of the STA graft were anastomosed to the left and right $\mathrm{A}_{3}$ segments (distal to the aneurysm) in an end-to-side manner, respectively. The occluding time for anastomosis was 25 and 20 minutes, respectively. The STA trunk then was anastomosed to the left $\mathrm{A}_{2}$ segment (proximal to the aneurysm) in an end-toside manner, with a 28-minute temporary occlusion (Fig. 4B). After completion of the anastomosis, the aneurysm was trapped along with the left ACA by an aneurysm clip. Then, a booster clip was applied to occlude the aneurysm to reconstruct the right ACA (Fig. 4C and D). Indocyanine green video angiography showed patency of the Y-shaped STA graft and occlusion of the aneurysm.

\section{Postoperative Course}

Postoperative diffusion-weighted MRI on the day after surgery revealed no ischemic or hemorrhagic complications (Fig. 5A). Angiography 6 months after surgery revealed disappearance of the aneurysm and patency of the Y-shaped STA graft (Fig. 5B and C). The postoperative course was uneventful, and the patient was discharged without any neurological deterioration.

\section{Discussion}

To the best of our knowledge, this is the first report of a de novo aneurysm that developed at the site of an ACAACA side-to-side anastomosis. A CFD study suggested
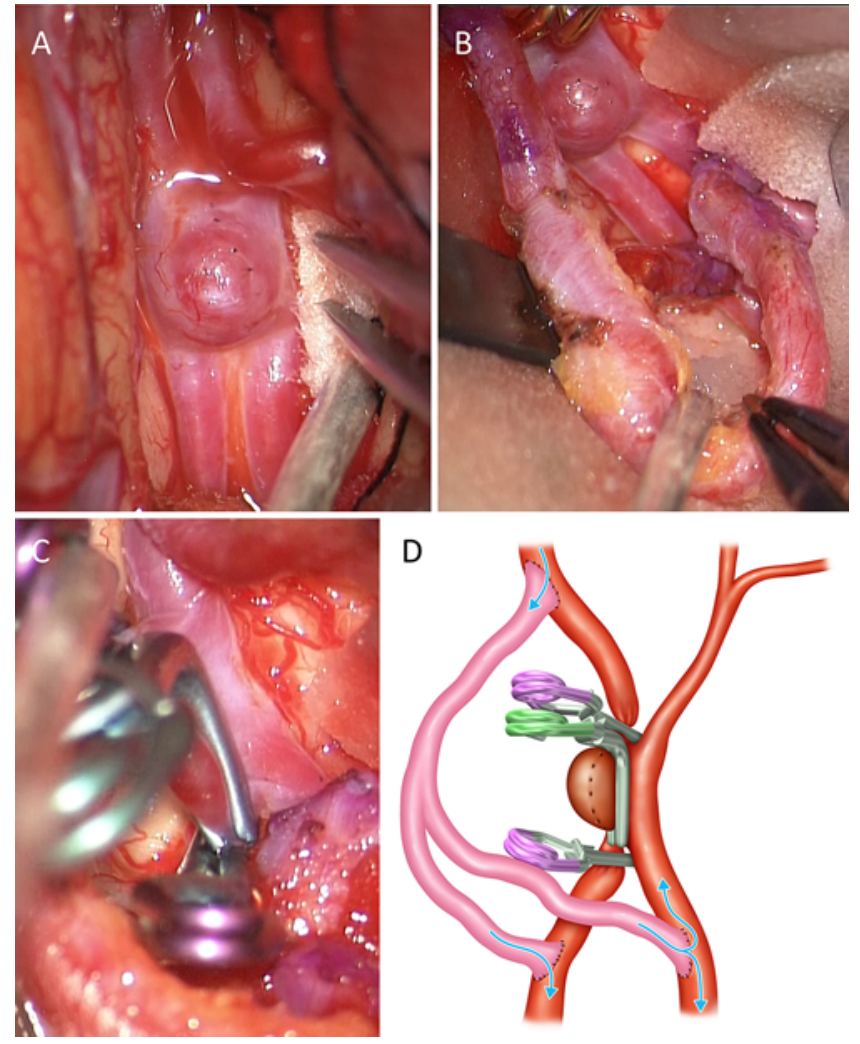

FIG. 4. A: Intraoperative photograph shows the aneurysm developed in the front wall of the anastomosis site, and projects to the anterosuperior direction. Sutures had been placed in the anterior wall of the aneurysm during the previous surgery. B: Intraoperative photograph shows completion of the bypass using a Y-shaped STA graft. C: Intraoperative photograph shows the completed trapping of the aneurysm. D: Operative illustration shows the site of the anastomosis and clip application in the second surgery. Copyright Hidenori Endo (panel D). Published with permission. Figure is available in color online only.

the association between the hemodynamic stress and aneurysm formation. The aneurysm was obliterated successfully with reconstruction of both ACAs by a Y-shaped STA interposition graft bypass.

\section{Mechanisms for De Novo Aneurysm Formation After Bypass Surgery}

A total of 16 cases with de novo aneurysm formation at the site of anastomosis after STA-middle cerebral artery (MCA) bypass have been reported in the literature. ${ }^{4-7,10-13,15,17,18,20,21,24}$ The interval between bypass surgery and detection of the aneurysm ranged from 2 weeks to 27 years. ${ }^{7,20}$ Nine of these 16 cases were detected at the time of rupture, $,, 5,7,10,11,17,18,21$ and these hemorrhagic events resulted in a poor or even fatal clinical outcome.,13 Thus, long-term follow-up is necessary to avoid this fatal complication, although its incidence is low. Mechanical arterial wall injuries, such as intraoperative vascular manipulation or excessive adventitial stripping, might be an underlying cause of aneurysm formation. ${ }^{13}$ In addition to these factors, recent studies have suggested that the increased hemodynamic stress leads to aneurysm formation because de novo aneurysms can arise from the MCA wall in an artificial T-shaped vasculature that is subjected to jet stream force 

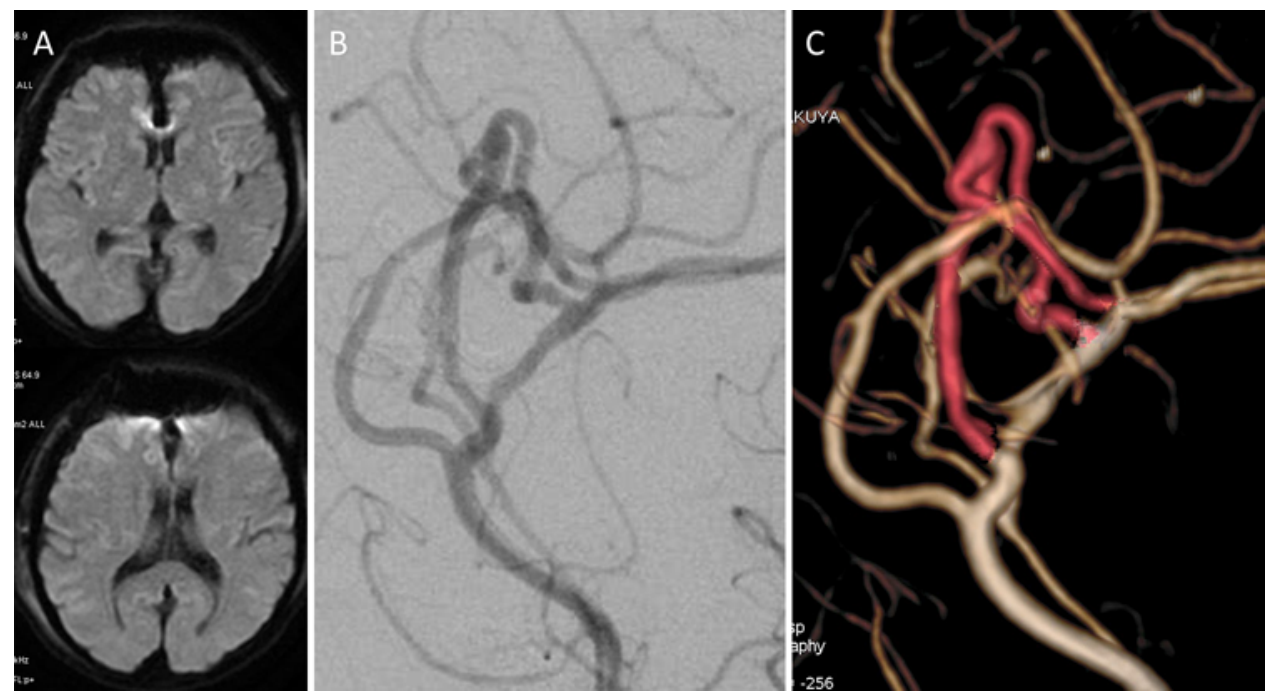

FIG. 5. A: Postoperative axial diffusion-weighted imaging obtained the day after surgery reveals no ischemic complications. B: Postoperative angiography 6 months after surgery shows obliteration of the aneurysm and patency of the Y-shaped STA graft. C: Three-dimensional angiography shows patency of the graft (pink). Figure is available in color online only.

from the STA. ${ }^{11,21,24}$ Based on this speculation, postoperative long-term control of blood pressure is important to reduce this hemodynamic stress. ${ }^{2,24}$ In our case, hairpinshaped blood flow was detected at the site of anastomosis in the DSA performed before aneurysm formation. This bloodstream might induce a localized region with high wall shear stress coincident with the pulsation in the front wall of the anastomosis site, where the aneurysm developed. These data suggested that the hemodynamic stress had a positive association with the aneurysm formation. Another possible explanation for aneurysm formation is inherent vascular fragility. It has been considered that patients with arterial dissection could have a constitutional, genetically determined weakness of the vessel wall and that environmental factors, such as acute infection or minor trauma, could act as triggers. ${ }^{3,22}$ The history of an intracranial dissecting aneurysm might have some association with the de novo aneurysm formation at the site of anastomosis, although genetic factors were not investigated.

\section{Revascularization for ACA}

Clipping, ${ }^{17,18,20,21}$ trapping with bypass, ${ }^{10}$ and wrapping $^{12,15}$ are used to treat a de novo aneurysm at the site of anastomosis. We considered the possibility that the whole vascular wall around the aneurysm was under a pathological condition, and the necessity to exclude this pathological part from the blood circulation as much as possible. One procedure to achieve this was simply to clip the aneurysm. However, clipping might cause a stenoocclusive complication because the aneurysm involved both ACAs. Thus, we performed revascularization of both ACAs using a Y-shaped STA interposition graft. We then performed trapping of the left ACA along with the aneurysm. The Yshaped STA graft supplied the blood flow to the bilateral ACAs: unidirectional flow in the left ACA and bidirectional flow in the right ACA, without ischemic complications. Another option to secure the aneurysm would be an endovascular approach. Stent-assisted coil embolization or flow diversion would be the potential solution. However, this aneurysm developed in the distal ACA, which was disadvantageous for an endovascular approach.

Several techniques have been reported for revascularization of bilateral ACAs. Sekhar et al..$^{23}$ used a radial artery graft from the MCA or STA to the $\mathrm{A}_{4}$ segment followed by $\mathrm{A}_{4}-\mathrm{A}_{4}$ side-to-side bypass, or a "Y" radial artery graft from the MCA or STA to both $\mathrm{A}_{4}$ segments. Inoue et al. ${ }^{8}$ reported a similar technique using a radial artery graft from the STA to the $\mathrm{A}_{3}$ segment followed by $\mathrm{A}_{3}-\mathrm{A}_{3}$ sideto-side anastomosis. Mirzadeh et al. ${ }^{14}$ reported the double reimplantation technique using a radial artery graft, which can connect the $\mathrm{A}_{2}$ segment to bilateral $\mathrm{A}_{3}$ segments. In contrast to these attractive but complicated techniques, Mura et al. ${ }^{16}$ reported a simple technique using a Y-shaped STA interposition graft, which is similar to the technique used in the present case. They performed revascularization for both pericallosal arteries with 3 end-to-end anastomoses during resection of the malignant meningioma with encasement of both pericallosal arteries. ${ }^{16}$ The use of a Y-shaped STA graft is advantageous because it can be harvested easily and quickly in the same surgical field, it needed only 3 sites of simple anastomosis, and the diameter was similar to that of the ACA. ${ }^{16}$ The disadvantage of the STA graft is delayed wound healing or necrosis. ${ }^{16}$ Furthermore, the STA can be a single trunk without the appropriate branch for a Y-shaped graft. It is necessary to harvest other grafts for this situation, such as the radial artery or saphenous vein. Preoperative assessment of the STA is necessary to determine whether the STA is appropriate as a graft.

\section{Conclusions}

De novo aneurysms rarely develop at the site of an ACA-ACA side-to-side anastomosis. A Y-shaped STA interposition graft is a useful option to treat this de novo aneurysm. Hemodynamic stress might be an underlying cause of aneurysm formation. Long-term follow-up is nec- 
essary to detect this rare complication after ACA-ACA anastomosis.

\section{Acknowledgments}

We would like to thank Enago for the English language review of our paper.

\section{References}

1. Abla AA, Lawton MT: Anterior cerebral artery bypass for complex aneurysms: an experience with intracranial-intracranial reconstruction and review of bypass options. J Neurosurg 120:1364-1377, 2014

2. Aoki T, Yoshitomi M, Yamamoto M, Hirohata M, Morioka M: Ruptured de novo aneurysm arising at a site remote from the anastomosis 14 years after superficial temporal arterymiddle cerebral artery bypass: a case report. Neurosurgery 71:E905-E909, 2012

3. Brandt T, Orberk E, Weber R, Werner I, Busse O, Müller BT, et al: Pathogenesis of cervical artery dissections: association with connective tissue abnormalities. Neurology 57:24-30, 2001

4. Eom KS, Kim DW, Kang SD: Intracerebral hemorrhage caused by rupture of a giant aneurysm complicating superficial temporal artery-middle cerebral artery anastomosis for moyamoya disease. Acta Neurochir (Wien) 152:1069-1073, 2010

5. Fein JM: Bypass induced cerebral aneurysm. Neurol Res 7:46-52, 1985

6. Fleischer AS, Faria MA Jr, Hoffmann JC Jr: Pseudoaneurysm complicating superficial temporal artery-middle cerebral artery bypass. Surg Neurol 12:305-306, 1979

7. Hokari M, Yasuda H, Iwasaki M, Kawabori M, Kuroda S, Abe S, et al: Intracerebral hemorrhage from a ruptured aneurysm at the site of anastomosis 27 years after superficial temporal artery-middle cerebral artery bypass. Neurol Med Chir (Tokyo) 50:1012-1014, 2010

8. Inoue T, Tsutsumi K, Ohno H, Shinozaki M: Revascularization of the anterior cerebral artery with an A3-A3 anastomosis and a superficial temporal artery bypass using an A3-radial artery graft to trap a giant anterior communicating artery aneurysm: technical case report. Neurosurgery 57:E207, 2005

9. Ito Z: [A new technique of intracranial interarterial anastomosis between distal anterior cerebral arteries (ACA) for ACA occlusion and its indication.] Neurol Med Chir (Tokyo) 21:931-939, 1981 (Jpn)

10. Kohno K, Ueda T, Kadota O, Sakaki S: Subdural hemorrhage caused by de novo aneurysm complicating extracranialintracranial bypass surgery: case report. Neurosurgery 38:1051-1055, 1996

11. Kurokawa T, Harada K, Ishihara H, Fujisawa H, Kato S, Kajiwara K, et al: De novo aneurysm formation on middle cerebral artery branches adjacent to the anastomotic site of superficial temporal artery-middle cerebral artery bypass surgery in two patients: technical case report. Oper Neurosurg 61 (Suppl 5):ONSE297-ONSE298, 2007

12. Lantos G, Fein JM, Knep S: Cortical artery aneurysm formation after extracranial to intracranial bypass surgery. Case report. J Neurosurg 60:636-639, 1984

13. Leclercq TA, Ambler MW: Fatal subdural bleeding following superficial temporal-middle cerebral artery anastomosis. Case report. J Neurosurg 52:392-394, 1980

14. Mirzadeh Z, Sanai N, Lawton MT: The azygos anterior cerebral artery bypass: double reimplantation technique for giant anterior communicating artery aneurysms. J Neurosurg 114:1154-1158, 2011

15. Miura Y, Kamei Y, Umeda Y, Shimosaka S: Disappearance of aneurysm complicating extracranial-intracranial bypass surgery: a case report. Surg Cereb Stroke 38:425-428, 2010

16. Mura J, Riquelme F, Cuevas JL, Luna F, Vizhnay P: Simplified azygos anterior cerebral bypass: $y$-shaped superficial temporal artery interposition graft from A2 with double reimplantation of pericallosal arteries: technical case report. Neurosurgery 72 (2 Suppl Operative):onsE235-onsE240, 2013

17. Nishimoto T, Yuki K, Sasaki T, Murakami T, Kodama Y, Kurisu K: A ruptured middle cerebral artery aneurysm originating from the site of anastomosis 20 years after extracranial-intracranial bypass for moyamoya disease: case report. Surg Neurol 64:261-265, 2005

18. Nishizawa S, Yokoyama T, Sugiyama K, Yokota N: Intracerebral hemorrhage from a ruptured pseudoaneurysm after STA-MCA anastomosis-case report. Neurol Med Chir (Tokyo) 40:408-412, 2000

19. Ota N, Tanikawa R, Miyama M, Matsumoto T, Miyazaki T, Matsukawa H, et al: Surgical strategy for complex anterior cerebral artery aneurysms: retrospective case series and literature review. World Neurosurg 87:328-345, 2016

20. Parent AD, Smith RR: Traumatic aneurysm complicating EC-IC bypass: successful surgical clipping. Surg Neurol 15:229-231, 1981

21. Sasaki T, Kodama N, Itokawa H: Aneurysm formation and rupture at the site of anastomosis following bypass surgery. Case report. J Neurosurg 85:500-502, 1996

22. Schievink WI: Spontaneous dissection of the carotid and vertebral arteries. N Engl J Med 344:898-906, 2001

23. Sekhar LN, Natarajan SK, Ellenbogen RG, Ghodke B: Cerebral revascularization for ischemia, aneurysms, and cranial base tumors. Neurosurgery 62 (6 Suppl 3):1373-1410, 2008

24. Yokota H, Yokoyama K, Noguchi H: De novo aneurysm associated with superficial temporal artery to middle cerebral artery bypass: report of two cases and review of literature. World Neurosurg 92:583.e7-583.e12, 2016

\section{Disclosures}

The authors report no conflict of interest concerning the materials or methods used in this study or the findings specified in this paper.

\section{Author Contributions}

Conception and design: H Endo. Acquisition of data: H Endo, Sugiyama. Analysis and interpretation of data: H Endo, Sugiyama. Drafting the article: H Endo, Sugiyama. Critically revising the article: T Endo. Reviewed submitted version of manuscript: $\mathrm{H}$ Endo, T Endo, Fujimura, Shimizu, Tominaga. Approved the final version of the manuscript on behalf of all authors: H Endo. Study supervision: Tominaga.

\section{Supplemental Information Videos}

Video 1. https://vimeo.com/230006445.

\section{Online-Only Content}

Supplemental material is available with the online version of the article.

Supplemental Methods. https://thejns.org/doi/suppl/10.3171/ 2017.6.JNS17931.

\section{Correspondence}

Hidenori Endo, Department of Neurosurgery, Kohnan Hospital, 4-20-1 Nagamachi-minami, Taihaku-ku, Sendai, Miyagi 9828523, Japan.email: hideendo@gmail.com. 